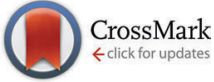

Cite this: J. Mater. Chem. B, 2016 4, 3094

Received 1st December 2015 Accepted 29th February 2016

DOI: $10.1039 / c 5 t b 02530 c$

www.rsc.org/MaterialsB

\section{Boronic acid-modified poly(amidoamine) dendrimers as sugar-sensing materials in water}

\author{
X. Liang and M. Bonizzoni*
}

Boronic acids can be used as receptors in chemical sensors for sugars, but their binding affinity and solubility are usually very poor in water. We improved these parameters by covalently connecting boronic acid moieties to the surface of third-generation poly(amido)amine (PAMAM) dendrimers to form a family of PAMAM-boronic acid receptors that display multivalent behavior. We confirmed the increased interaction strength of these modified boronic acid receptors using diol-containing dyes such as 4-methylesculetin and alizarin red $S$ as probes in optical spectroscopy experiments. We then translated these results to sugar sensing using the self-assembled [PAMAM-ba.(dye) ${ }_{n}$ ] complexes as sensors in an indicator displacement assay. Our approach successfully detected simple sugars (e.g. fructose, glucose, galactose and ribose) in water, traditionally a very challenging medium for carbohydrate detection. Finally, we demonstrated the use of these polymer-based sensors in a multivariate array sensing platform for the discrimination of simple sugars in water as a proof of principle towards their broader applicability under physiologically and environmentally relevant conditions.

\section{Introduction}

An ongoing quest of sensing science is to create an efficient sensing method to detect and discriminate sugars with good sensitivity and selectivity. ${ }^{1-6}$ Sugars play crucial roles as carbon and energy sources to build and fuel cells in living creatures; carbohydrate conjugates are also crucially involved in cell signalling and recognition in living systems. ${ }^{7-9}$ They relate to most of the important functioning processes in our bodies such as controlling metabolism, stress resistance, growth and development. ${ }^{10}$ In biological systems, enzymes are used to sense and recognize different sugars with a high selectivity due to their unique geometric shapes.

Although sugars have also long been established as important targets for which efficient artificial sensors are required, ${ }^{11}$ sugar sensing is still challenging using synthetic receptors. First, many synthetic receptors do not dissolve well in aqueous solution, in which sugars dissolve. Second, sugars have high solvation enthalpies in water that are difficult for synthetic sensors to overcome. Third, selectivity has been generally difficult to achieve as most simple sugars show very little structural difference except configurations of certain stereocenters. ${ }^{12}$

The covalent reversible interaction of boronic acids with diols (shown in Scheme 1) has long generated great interest in this field as one of the few chemical interactions that are

Department of Chemistry, The University of Alabama, Box 870336, Tuscaloosa, AL 35487-0336, USA. E-mail: marco.bonizzoni@ua.edu

$\dagger$ Electronic supplementary information (ESI) available. See DOI: 10.1039/c5tb02530c

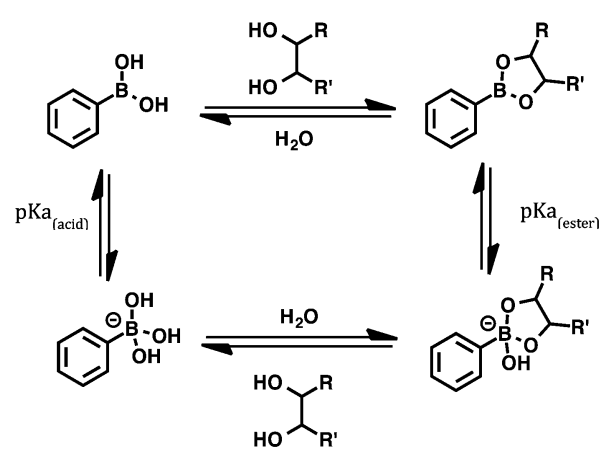

Scheme 1 The boronic acid-diol interaction at low and high $\mathrm{pH}$ in water.

somewhat selective for sugars under highly competitive conditions; this property has allowed the development of boronic acid-based chemosensors to detect sugars functioning in organic solvents, but the relatively low affinity associated with this process in water has made direct sensing in this crucial medium extremely challenging. ${ }^{13}$

Two classical methods, direct sensing and indicator displacement assays (IDA), have been used to design sugar sensing systems (Scheme 2). In a direct sensing approach, a signalling unit is covalently and permanently connected to a recognition unit; binding of the analyte of interest to the recognition unit results in a signal change. In sugar sensing, the recognition unit often contains a boronic acid moiety; signalling is commonly accomplished by exploiting a change in the absorption or emission properties of an appropriately chosen chromophore. Successful boronic acid sugar receptors used in direct sensing are often 

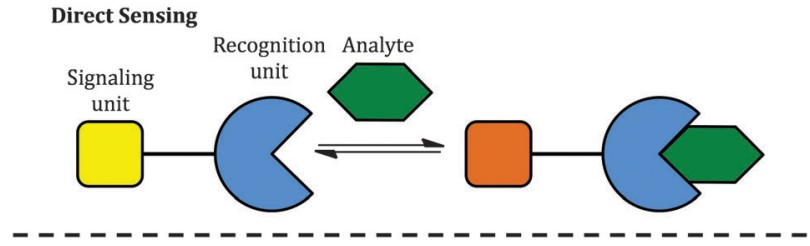

Indicator Displacement Array (IDA)

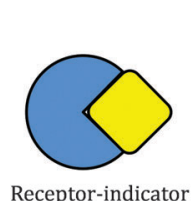

complex
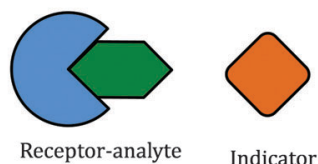

complex

Indicator

Scheme 2 Two approaches to the construction of chemical sensors: (top) direct sensing; (bottom) an indicator displacement assay (IDA).

multivalent in order to overcome the high solvation enthalpies of sugars in water. ${ }^{14-16}$ To increase the solubility of such receptors in water, the boronic acids have been covalently linked to water-soluble polymers, ${ }^{17}$ or bound to a charged moiety. ${ }^{18}$ Selectivity has been typically difficult to achieve. ${ }^{18,19}$

However, one disadvantage of direct sensing is that the selectivity of the sensing system is difficult to tune since the signalling unit and the recognition unit are covalently linked. Besides, higher synthetic effort goes into creating a receptor compared to IDA. In the latter case, the recognition and signalling units are separate molecules, held together by noncovalent interactions to form a supramolecular sensor; upon exposure to analytes, the signalling unit is then displaced by the analyte, leading to a detectable signal change (Scheme 2). The properties of an IDA sensing system can be tuned more easily because of the increased flexibility afforded by the noncovalent linkage. IDA systems have been proposed for sugar sensing as well: for instance, a complex between phenylboronic acid and dye alizarin red S (ARS) has been used to sense sugars, but the relatively low interaction energy limited the applicability of this system to organic solvents. ${ }^{20}$ However, there is no single system able to sense different sugars effectively.

Here we report on our efforts towards the creation of a sugarsensitive boronic acid-modified water-soluble polymeric material capable of sugar detection and discrimination in neat water solution. Sensing is achieved using an IDA approach based on two synthetically modified boronic acid-PAMAM receptors (PAMAM-m-ba and PAMAM-o-ba) and two commercially available dyes (ML and ARS), at two pH levels (7.4 and 10.0). As further proof of the functionality of these receptors, we also report on the development of an array-sensing system based on these receptors capable of discriminating between four common sugars (fructose, glucose, galactose, and ribose) in neat water.

We selected poly(amidoamine) (PAMAM) dendrimers ${ }^{21,22}$ as polymeric scaffolds with which to build our multivalent sugarreactive sensors because of their excellent water solubility over a wide range of $\mathrm{pH}$ values, ${ }^{23,24}$ their highly branched, globular and symmetrical structure, ${ }^{25}$ the large number of surface groups available for covalent surface modification, and their wide commercial availability in high purity. By decorating the surface
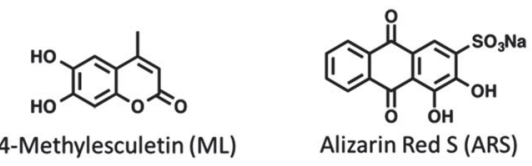

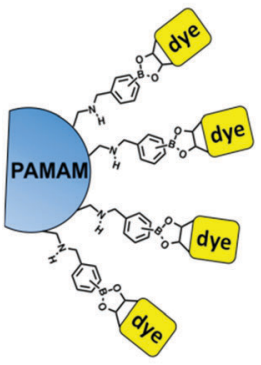

[PAMAM-ba $\cdot$ dye]

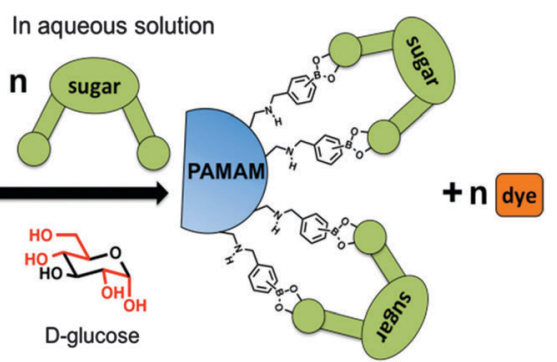

[PAMAM-ba • sugar]
Scheme 3 Top: Chemical structures of dyes 4-methylesculetin (ML) and alizarin red S (ARS). Bottom: A sugar molecule displaces multiple dye molecules bound to the surface of the PAMAM-ba receptors in an indicator displacement sensing paradigm.

of a PAMAM dendrimer with boronic acids, we aimed to significantly increase the solubility of the boronic acid recognition moieties and to introduce beneficial multivalency in the receptors: multiple interactions of the receptor with each analyte will afford higher overall binding affinity and a much better chance for these sensors to work even in neat water, being able to overcome energetic barrier posed by the high cost of desolvation of these sugar analytes in aqueous media.

We then selected dyes 4-methylesculetin (ML) and alizarin red S (ARS) (see Scheme 3) for the construction of an indicator displacement assay. The Anslyn ${ }^{26-28}$ and Wang groups ${ }^{20,29}$ have previously reported on the binding of these dyes to boronic acid moieties accompanied by changes in the absorbance spectrum and enhanced fluorescence emission.

We constructed sugar sensors for use in an IDA scheme by forming complexes of these two dyes with our surface-modified PAMAM dendrimers ([PAMAM-ba-dye], Scheme 3). When an analyte (sugar) is added to this complex, dye molecules are displaced by the sugar; because the dye displays characteristically different spectroscopic signatures when free or bound, the dye's optical signal reports on the binding of the sugar guest (Scheme 3, bottom).

\section{Experimental}

The following numbering scheme will be used for the assignment of ${ }^{1} \mathrm{H}$-NMR spectra in these polymers: $\left({ }^{\mathrm{a}} \mathrm{CH}_{2}{ }^{\mathrm{a}} \mathrm{CH}_{2}\right)\left[\mathrm{N}\left({ }^{\mathrm{b}} \mathrm{CH}_{2}\right.\right.$ ${ }^{\mathrm{c}} \mathrm{CH}_{2} \mathrm{CONH}^{\mathrm{d}} \mathrm{CH}_{2}{ }^{\mathrm{e}} \mathrm{CH}_{2} \mathrm{~N}\left({ }^{\mathrm{f}} \mathrm{CH}_{2}{ }^{\mathrm{g}} \mathrm{CH}_{2} \mathrm{CONH}^{\mathrm{h}} \mathrm{CH}_{2}{ }^{\mathrm{i}} \mathrm{CH}_{2} \mathrm{~N}\left({ }^{\mathrm{j}} \mathrm{CH}_{2}{ }^{\mathrm{k}} \mathrm{CH}_{2}\right.\right.$ $\mathrm{CONH}^{\mathrm{l}} \mathrm{CH}_{2}{ }^{\mathrm{m}} \mathrm{CH}_{2} \mathrm{~N}\left({ }^{\mathrm{n}} \mathrm{CH}_{2}{ }^{\mathrm{o}} \mathrm{CH}_{2} \mathrm{CONH}^{\mathrm{P}} \mathrm{CH}_{2}{ }^{\mathrm{q}} \mathrm{CH}_{2} \mathrm{NH}^{\mathrm{r}} \mathrm{CH}_{2} \mathrm{C}_{6} \mathrm{H}_{4} \mathrm{~B}\right.$ $\left.\left.\left.\left.\left.(\mathrm{OH})_{2}\right)_{2}\right)_{2}\right)_{2}\right)_{2}\right]_{2}$. Also see the $\mathrm{ESI} \dagger$ for full spectra, assignments, and further details.

\section{PAMAM- $m$-ba}

To a $25 \mathrm{~mL}$ round bottom flask were added a $20 \mathrm{wt} \% \mathrm{MeOH}$ solution of the ethylenediamine core $(1.00 \mathrm{~g})$, amine terminated third-generation PAMAM dendrimers (0.0289 mmol), $0.174 \mathrm{~g}$ (1.16 mmol) of 3-formylphenylboronic acid, and $1.30 \mathrm{~g}$ of freshly 
regenerated $4 \AA$ molecular sieves. The mixture was stirred at $70{ }^{\circ} \mathrm{C}$ in an oil bath for 3 days. The molecular sieves were then removed by vacuum filtration and washed $3 \times$ with $\mathrm{MeOH}$. The washing solvent was returned to the reacting mixture. To this mixture were added $0.665 \mathrm{~g}(17.6 \mathrm{mmol})$ of $\mathrm{NaBH}_{4}$ and $3.5 \mathrm{~mL}$ of $\mathrm{MeOH}$. The resulting mixture was stirred at r.t. for $24 \mathrm{~h}$. Solvent was then removed on a rotary evaporator, yielding a solid, which was re-dissolved in $6.0 \mathrm{~mL}$ of a $1: 1$ mixture of $\mathrm{H}_{2} \mathrm{O}$ and $\mathrm{MeOH}$. The solution was transferred to a dialysis membrane (6-8 kDa MW cut-off), which was sealed and suspended in $800 \mathrm{~mL}$ of a $1: 1 \mathrm{MeOH}: \mathrm{H}_{2} \mathrm{O}$ mixture for $3 \mathrm{~h}$. The membrane was then removed from this mixture and suspended in $800 \mathrm{~mL}$ of neat DI water for $24 \mathrm{~h}$, followed by $800 \mathrm{~mL}$ of neat $\mathrm{MeOH}$ for another $24 \mathrm{~h}$. The solution in the membrane was collected in a tared $50 \mathrm{~mL}$ round bottom flask, and the volatiles were removed on a rotary evaporator, yielding $0.221 \mathrm{~g}$ of a colourless solid (68.3\%).

${ }^{1} \mathrm{H}-\mathrm{NMR}\left(360 \mathrm{MHz}, \mathrm{CD}_{3} \mathrm{OD}, \mathrm{ppm}\right) \delta_{\mathrm{H}}: 7.40-7.60(50 \mathrm{H}$, $\left.o-\mathrm{PhB}(\mathrm{OH})_{2}\right), 7.22-7.32\left(25 \mathrm{H}, p-\mathrm{PhB}(\mathrm{OH})_{2}\right), 7.05-7.20 \quad(25 \mathrm{H}$, $\left.m-\mathrm{PhB}(\mathrm{OH})_{2}\right), 4.58(50 \mathrm{H}, \mathrm{r}), 3.51-3.77(120 \mathrm{H}, \mathrm{d}, \mathrm{h}, \mathrm{l}, \mathrm{p}), 2.63-$ 3.00 (184H, b, f, j, n, q), 2.49-2.63 (58H, a, e, i, m), 2.04-2.49 $(120 \mathrm{H}, \mathrm{c}, \mathrm{g}, \mathrm{k}, \mathrm{o})$. MALDI-TOF: calcd for $\mathrm{C}_{477} \mathrm{H}_{783} \mathrm{~N}_{122} \mathrm{O}_{110} \mathrm{~B}_{25} \mathrm{Na}=$ $[\mathrm{M}+\mathrm{Na}]^{+}:$10279.2. Found: 10279.0 .

\section{PAMAM-o-ba}

To a $25 \mathrm{~mL}$ round bottom flask were added a 20 wt $\%$ $\mathrm{MeOH}$ solution of the en-core $(1.00 \mathrm{~g})$, amine terminated G3 PAMAM dendrimers $(0.0289 \mathrm{mmol}), 0.174 \mathrm{~g}(1.16 \mathrm{mmol})$ of 2-formylphenylboronic acid, and $1.45 \mathrm{~g}$ of freshly regenerated $4 \AA$ A molecular sieves. The mixture was stirred at a $70{ }^{\circ} \mathrm{C}$ in an oil bath for 3 days. The molecular sieves were then removed by vacuum filtration and washed $3 \times$ with $\mathrm{MeOH}$. The washing solvent was returned to the reaction mixture, to which $1.34 \mathrm{~g}$ (35.42 mmol) of $\mathrm{NaBH}_{4}$ and $6.0 \mathrm{~mL}$ of $\mathrm{MeOH}$ were added. The resulting mixture was left to stir at r.t. for $24 \mathrm{~h}$. The solvent was removed on a rotary evaporator to yield a solid, which was re-dissolved in $6.0 \mathrm{~mL}$ of $\mathrm{MeOH}$. This solution was transferred to a rehydrated dialysis membrane (6-8 kDa MW cut-off), which was suspended in $800 \mathrm{~mL}$ of a $1: 1 \mathrm{MeOH}: \mathrm{H}_{2} \mathrm{O}$ mixture for $24 \mathrm{~h}$. The membrane was then transferred to $800 \mathrm{~mL}$ of neat DI water for another $24 \mathrm{~h}$, and finally to $800 \mathrm{~mL}$ of neat $\mathrm{MeOH}$ for $24 \mathrm{~h}$. The solution was then collected in a $25 \mathrm{~mL}$ tared round bottom flask and the volatiles were evaporated to yield $0.0973 \mathrm{~g}(30.2 \%)$ of a colourless solid.

${ }^{1} \mathrm{H}-\mathrm{NMR}\left(360 \mathrm{MHz}, \mathrm{CD}_{3} \mathrm{OD}, \mathrm{ppm}\right) \delta_{\mathrm{H}}: 7.33-7.50(26 \mathrm{H}$, $\left.o-\mathrm{PhB}(\mathrm{OH})_{2}\right), 7.16-7.25\left(52 \mathrm{H}, m-\mathrm{PhB}(\mathrm{OH})_{2}\right), 7.09-7.16(26 \mathrm{H}$, p-PhB(OH $\left.)_{2}\right), 4.04$ (52H, r), 3.45-3.59, (56H, d, h, l), 3.15-3.25 (64H, p), 2.90-3.13 (64H, q), 2.72-2.81 (120H, b, f, j, n), 2.49-2.53 (58H, a, e, i, m), 2.27-2.41 (120H, c, g, k, o). MALDI-TOF: calcd for $\mathrm{C}_{484} \mathrm{H}_{790} \mathrm{~N}_{122} \mathrm{O}_{112} \mathrm{~B}_{26} \mathrm{~K}=[\mathrm{M}+\mathrm{K}]^{+}$: 10429.2. Found: 10429.0.

\section{Titration conditions and multivariate analysis}

See ESI. $\dagger$

\section{Results and discussion}

\section{Design and synthesis of the polymeric receptors}

Commercially available amine-terminated PAMAM dendrimers are ideal starting scaffolds to construct large yet compact and globular multivalent structures in which multiple binding sites are held in close proximity to each other, facilitating multiple interactions with their intended guest, and ultimately opening the way for a significant affinity boost. The fact that these polymers are commercially available in high purity, ${ }^{30}$ and that they possess reactive amine surface groups that can be selectively functionalized with a host of standard techniques significantly adds to their value in this application. ${ }^{31-33}$ James and Shinkai previously reported on the modification with boronic acids of a branched polymer equivalent in size to a G1 PAMAM dendrimer. In that case they fully converted the primary amine termini, which were also decorated with a fluorophore for detection. The much smaller receptor they obtained did display increased affinity towards simple sugars over a plain boronic acid, but unfortunately it only worked in neat $\mathrm{MeOH}$, as it was insoluble in water. $^{34}$

In the approach presented here, we used the PAMAM dendrimers' high water solubility across a wide $\mathrm{pH}$ range to improve the solubility and water-compatibility of the boronic acid sensing moieties. To do so, we specifically sought incomplete conversion of the dendrimer's terminal groups into boronic derivatives, so that some of the unreacted surface amine groups in these receptors can be protonated in water solution, and the resulting positive charges will improve the solubility of our receptor under these conditions. In this context, a larger third generation (G3) PAMAM dendrimer provides an excellent compromise between achieving a large enough size to obtain a significant affinity boost and yet to be affordable and practical enough for other researchers to adopt our method.

We synthesized two receptors, one with the boronic acid group in the ortho position to the PAMAM attachment point (PAMAM-o-ba), and the other with attachment at the meta position (PAMAM-m-ba). Previous studies on ( $p$-methylamino)phenylboronic acids have shown that the substitution pattern is ineffective in binding to diol moieties, so we did not pursue it here. ${ }^{35,36}$ Covalent modification of the dendrimers was achieved in a two-step, one-pot reaction involving the formation of an imine by reaction of the primary amines on the dendrimer's surface with formyl groups of commercially available formyl phenylboronic acids, followed by in situ reduction to the corresponding secondary amine. This pathway (Scheme 4) afforded the final products in good yields and purity.

The reaction was conveniently monitored by ${ }^{1} \mathrm{H}-\mathrm{NMR}$ to follow the degree of conversion of the starting aldehyde (signal at 10.0-10.1 ppm) into the corresponding imine (signal at 8.0$9.5 \mathrm{ppm}$ ). When sufficient conversion was achieved as judged by NMR spectroscopy, the condensation reaction was interrupted by introduction of a large excess of $\mathrm{NaBH}_{4}$, which both reduced the newly formed imine to the desired secondary amines, and inactivated the excess of formyl phenylboronic acid by reduction of its carbonyl moiety. ${ }^{37-39}$ 

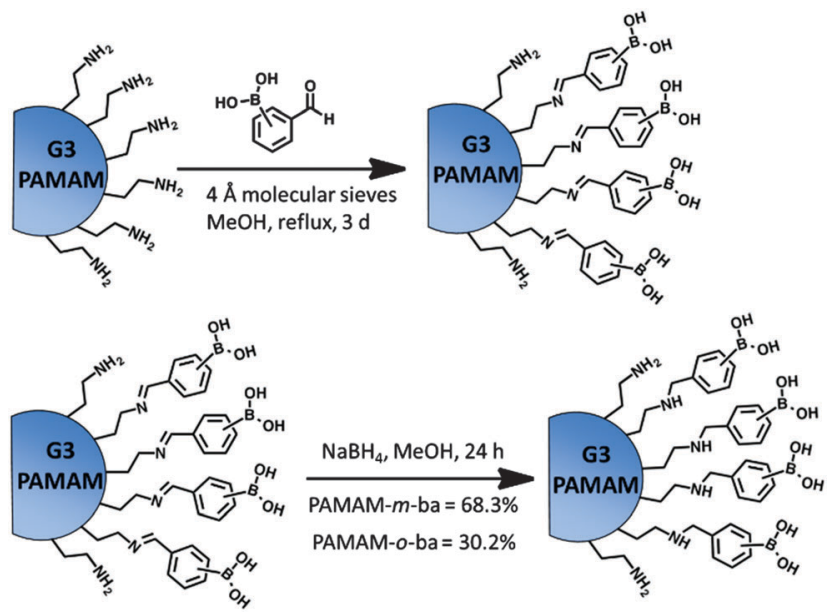

Scheme 4 Synthesis of receptors PAMAM- $\boldsymbol{m}$-ba and PAMAM-o-ba. Isolated yields after purification by membrane dialysis were $68.3 \%$ and $30.2 \%$ respectively.

The polymeric product was then purified by repeated equilibrium dialysis in alternating water and methanol to remove the excess small-molecule reagents and inorganic by-products. After a final solvent exchange to $\mathrm{MeOH}$, the each PAMAM-ba product was obtained as a solid after evaporation of the organic solvent. The purity of the product was confirmed by ${ }^{1} \mathrm{H}$ NMR and MALDITOF spectra, which allowed us to calculate an average extent of conversion of the surface amines to boronic acid groups; conversion was found to be around $80 \%$ for both receptors, in accordance with our partial conversion goal stated above. Since G3 PAMAM dendrimers has totally 32 branches, around 25 of those ended up carrying boronic acid moieties.

We will show below that these water-soluble multivalent receptors are able to bind to the diol functional group efficiently, and that they can be used in the construction of effective IDA sensors for sugars in neat water.

\section{PAMAM-ba binding to the 4-methylesculetin (ML) dye}

Emission. We first studied the binding of 4-methylesculetin (ML) to our boronic acid modified dendrimers in water at controlled $\mathrm{pH}$. In this connection, the Anslyn group reported that the fluorescence intensity of the ML dye (see Scheme 3) was typically found to increase upon binding to a boronic acid moiety. ${ }^{28} \mathrm{We}$ conducted similar binding studies by adding aliquots of PAMAM$\boldsymbol{m}$-ba to a solution of ML in water at buffered neutral $\mathrm{pH}$ (7.4). We initially observed a significant decrease in the fluorescence intensity, followed by a revival of the fluorescence as more PAMAM- $\boldsymbol{m}$-ba was added, and ultimately the intensity of the fluorescence emission did indeed increase well past its starting point, as expected. These results are reported in Fig. 1 below; note that throughout this work the measured properties are plotted as a function of the effective calculated concentration of boronic acid moieties in the solution (referred to as $[m$-ba $]$ or $[o$-ba $]$ in the plots), rather than as the total concentration of modified PAMAM receptor.

The quenching observed initially may be due to two possible effects. On the one hand, at the beginning of the titration a

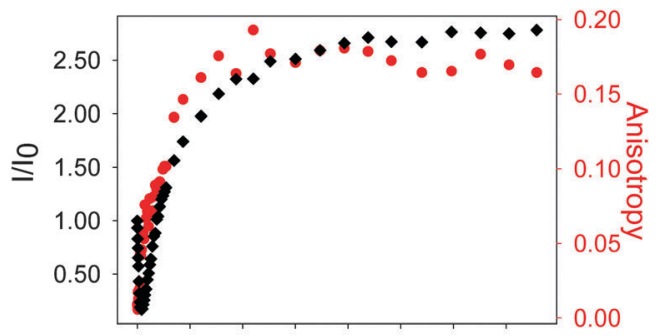

$\begin{array}{llllllll}0.0 & 0.5 & 1.0 & 1.5 & 2.0 & 2.5 & 3.0 & 3.5\end{array}$

[m-ba] (mM)

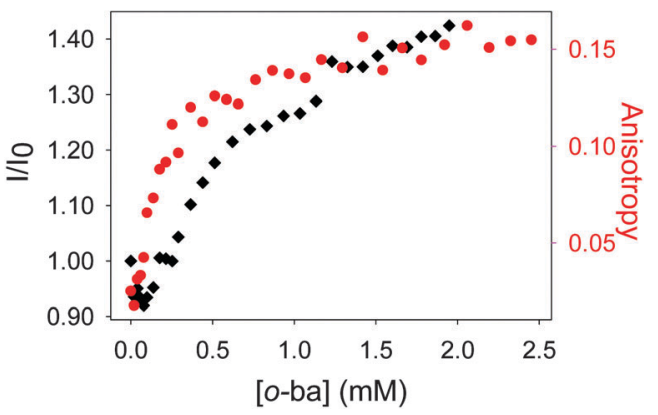

Fig. 1 Normalized fluorescence intensity and anisotropy changes as PAMAM- $\boldsymbol{m}$-ba (top) and PAMAM-o-ba (bottom) were added to ML aqueous solutions. $T=25^{\circ} \mathrm{C} ; \mathrm{pH}=7.4$ in $50 \mathrm{mM}$ HEPES; $[\mathrm{ML}]=1.057 \times 10^{-5} \mathrm{M}$; $\lambda_{\text {exc }}=362 \mathrm{~nm} ; \lambda_{\text {em }}=450 \mathrm{~nm}$.

large excess of ML dye is present with respect to the polymer, so high molecularity dye-polymer complexes will be formed, which may lead to self-quenching of the dye as its molecules are forced into close proximity. However, this is expected to be a negligible effect for a dye like ML, which displays the typical large Stokes shift associated with coumarin-type fluorophores. Instead, it is more likely that two processes are at work simultaneously in this interaction: inefficient dynamic quenching of ML by random collision of excited ML molecules with boronic acids on the surface of the dendrimer, and more efficient fluorescence reviving upon formation of the ground-state complex between ML and a boronic acid moiety. As more polymer is added to the solution during the course of the titration the extent of binding increases, making the latter process more important and causing an overall increase in the observed fluorescence intensity.

Anisotropy. This hypothesis was confirmed by monitoring the fluorescence anisotropy signal through the course of the same titration. Fluorescence anisotropy reports on the degree of tumbling of fluorophores in solution, so it is a faithful and effective report of the degree of binding in the interaction of a small fluorophore binding to a large polymer, because the free and bound fluorophore molecules have starkly different rates of tumbling, i.e. very high and very low, respectively. Indeed, the steady increase in the anisotropy signal (in red in Fig. 1) shows that ML is in fact binding to the receptor from the very start of the titration, so the non-monotonous trend in the fluorescence emission can safely be ascribed to the conflicting quenching and reviving processes described above.

Binding affinity determinations. The anisotropy signal described above was used to determine the binding constant 


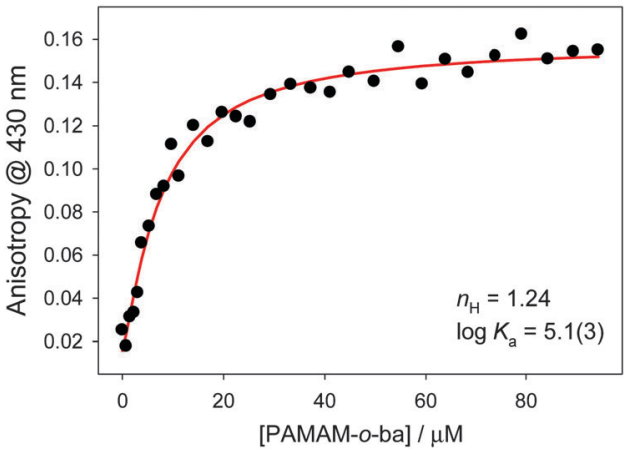

Fig. 2 Binding isotherm obtained from addition of PAMAM-o-ba to ML. The experimental data were fit to a modified Hill isotherm (red) $r=\left(r_{\max }-\right.$ $\left.r_{\text {min }}\right)$ [PAMAM-o-ba $]^{n} /\left([\text { PAMAM-o-ba }]^{n}+\left(1 / K_{\mathrm{a}}\right)^{n}\right)+r_{\min } \cdot T=25^{\circ} \mathrm{C} ; \mathrm{pH}=7.4$ in water (50 mM HEPES); $[M L]=1.027 \times 10^{-5} \mathrm{M} ; \lambda_{\mathrm{exc}}=362 \mathrm{~nm} ; \lambda_{\mathrm{em}}=450 \mathrm{~nm}$.

between the PAMAM-ba hosts and the ML guest, by fitting the experimental results to a modified Hill model accounting explicitly for multivalency. In the case of PAMAM-o-ba an association constant $\log K_{\mathrm{a}}=5.1(3)$ was determined, as shown in Fig. 2.

This value was compared to that obtained from the binding of isolated 2-formylphenylboronic acid to the ML dye, for which we calculated a $\log K_{1: 1}=3.61(3)$ (see ESI $\dagger$ ), which is in accordance with literature reporting that boronic acids decorated in their ortho position display significant affinity for diols. ${ }^{28}$ Indeed, 2-formylphenylboronic acid was the only isomeric boronic acid that formed complexes with this dye (see ESI $\dagger$ ). Nevertheless, the much higher binding constant reported for the boronic acid site in the dendritic structure $v s$. the isolated one strongly supports our hypothesis that inclusion of the boronic acid moiety in the PAMAM structure significantly increases its affinity for diols.

The binding affinity of PAMAM- $\boldsymbol{m}$-ba for the ML dye was also determined in the same way $\left(\log K_{\mathrm{a}}=5.0(3)\right)$; unfortunately, however, the corresponding 3-formylphenylboronic acid did not display any binding interaction with ML (see discussion below), so a direct comparison was not possible in that case.

The origin of quenching. We separately confirmed that isolated boronic acids are capable of quenching the ML dye through a dynamic quenching process. Namely, aliquots of a solution of simple 3-formylphenylboronic acid were added to an ML solution during the course of a titration. As seen from Fig. 3, the fluorescence intensity decreased as 3-formylphenylboronic acid was added. The data were an excellent fit to the Stern-Volmer quenching model, which confirmed the presence of dynamic quenching in this case. ${ }^{40}$

It is also noteworthy that no binding was observed between ML and this simple isolated boronic acid, although binding of ML to the polymeric PAMAM-ba receptors was clearly observed under comparable conditions (see Fig. 1). This further highlights the large increase in affinity.

\section{PAMAM-ba binding to alizarin red S (ARS) dye}

Emission. The Wang group reported that alizarin red S (ARS, see Scheme 3) also binds to isolated boronic acids; in this case
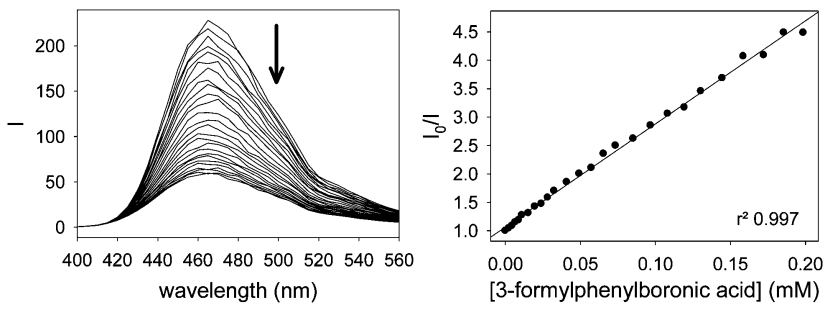

Fig. 3 The $\mathbf{M L}$ dye is quenched by 3-formylphenylboronic acid. Left: Emission spectra; right: Stern-Volmer plot and the corresponding linear fit. $T=25^{\circ} \mathrm{C} ; \mathrm{pH}=7.4$ in water $(50 \mathrm{mM} \mathrm{HEPES}) ;[\mathrm{ML}]=1.057 \times 10^{-5} \mathrm{M}$; $\lambda_{\mathrm{exc}}=362 \mathrm{~nm} ; \lambda_{\mathrm{em}}=450 \mathrm{~nm}$.

the dye displays very little fluorescence when free, with the emission increasing significantly upon binding. ${ }^{20,29}$ We observed a similar behaviour with our polymeric PAMAM-ba receptors: as aliquots of each receptor were added to a buffered aqueous solution of ARS, the fluorescence was very strongly enhanced (Fig. 4), until a plateau was reached indicating the complete binding of the dye present in solution. The results for PAMAM$\boldsymbol{m}$-ba are reported in Fig. 4 below; comparable results were obtained for PAMAM-o-ba (see below and ESI $\dagger$ ).

Anisotropy. Unfortunately, in this case anisotropy measurements were not informative: increasingly high values compatible with the formation of scattering particles were observed during the course of the titration, which completely overshadowed the binding signal. We hypothesize that although both PAMAM-m-ba and ARS are soluble in water at the concentrations involved, the [PAMAM- $\boldsymbol{m}$-ba-ARS] complex is not.

Light scattering. To confirm this, we used dynamic light scattering (DLS) to monitor the time evolution of a solution containing ARS with a sufficient concentration of PAMAM-m-ba to induce complete binding of the dye. Indeed, DLS measurements performed as soon as possible after mixing of the two binding partners indicated the formation of particles with the hydrodynamic diameter estimated to be around $590 \pm 30 \mathrm{~nm}$; as the solution aged over the course of multiple hours, the average particle size steadily increased; when the experiment was terminated the mixture was clearly hazy to the naked eye. Aggregates of such size are sure to cause very significant scattering, which in turn hampers fluorescence anisotropy measurement. A similar experiment with the ML dye did not detect the formation of aggregates.

The formation of nanoscopic aggregates has been previously observed for these polyelectrolytes in water solution. Indeed, PAMAM dendrimers and other similar polyelectrolytes have
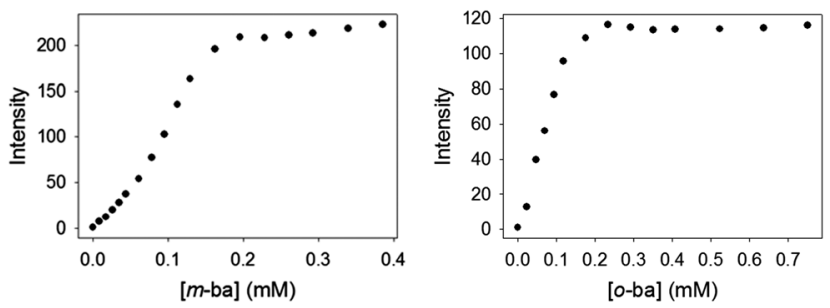

Fig. 4 Binding titration of ARS dye with PAMAM- $m$-ba (left) and PAMAMo-ba (right) monitored by fluorescence emission intensity. $T=25^{\circ} \mathrm{C} ; \mathrm{pH}=$ 7.4 in $50 \mathrm{mM} \mathrm{HEPES}$; [ARS] $=5.257 \times 10^{-5} \mathrm{M} ; \lambda_{\mathrm{exc}}=518 \mathrm{~nm} ; \lambda_{\mathrm{em}}=545 \mathrm{~nm}$. 
been shown to form nanoparticles of definite and controllable size and shape, and assembly triggering was demonstrated with polyhydroxylated compounds such as cyclodextrins. ${ }^{41,42}$ Both electrostatic effects and solvation changes have been deemed responsible for the formation of aggregates displaying decreased solubility. ${ }^{43}$ These reports strongly support our hypothesis that the dendrimers themselves are soluble under the conditions of our work, but the dendrimer-ARS complexes exhibit decreased solubility.

Absorbance. Fortunately, however, this process did not prevent us from monitoring the absorption properties of the solution, so that we were able to monitor the binding process by UV-Vis spectroscopy as well as by fluorescence emission. As seen from Fig. 5, the absorption of ARS displays a blue shift as each receptor is added.

Kinetics. A result consistent with the DLS determination was observed by following the kinetics of the aggregation process with the ARS dye using UV-Vis spectroscopy. The results (Fig. 6) show that the baseline absorption increased as time passed, indicating that the [PAMAM-m-ba-ARS] complex gradually aggregated and formed nanoparticles in aqueous solution. Under these conditions, it is difficult to reach an equilibrium state, if at all possible. We therefore had to take this effect into careful consideration when carrying out further experiments, by making sure that additions and waiting times were carefully timed and repeated exactly when an experiment was reproduced.
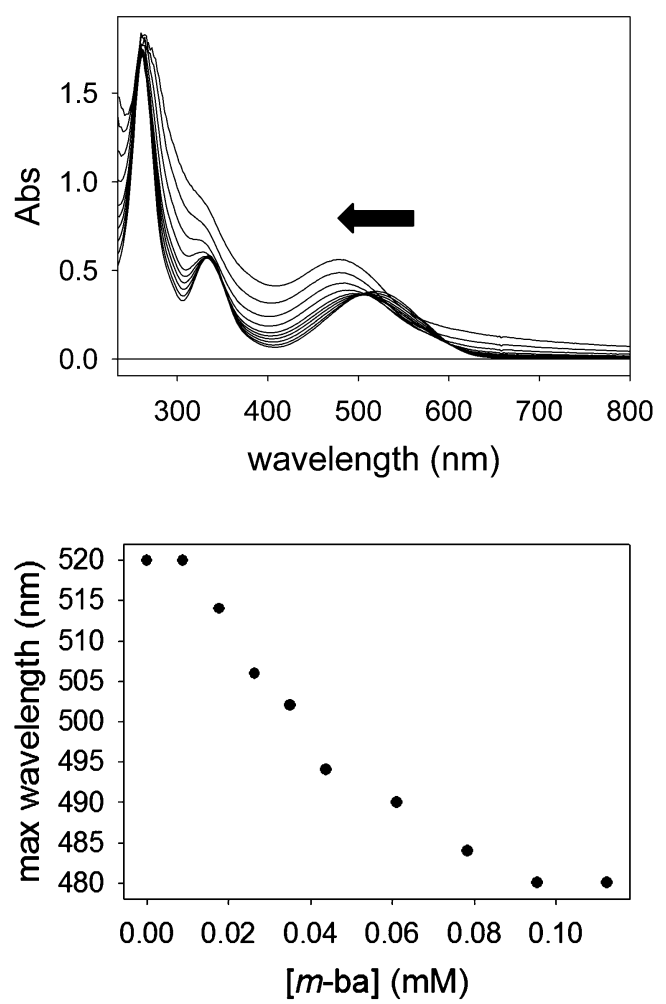

Fig. 5 (top) A blue shift in the absorbance spectrum of an aqueous solution of ARS is observed as PAMAM- $\boldsymbol{m}$-ba is added. (bottom) The position of the absorption maximum at around $500 \mathrm{~nm}$ as a function of the added polymer. $T=25^{\circ} \mathrm{C}$; pH $=7.4$ in $50 \mathrm{mM} \mathrm{HEPES} ;[$ ARS $]=5.208 \times 10^{-5} \mathrm{M}$.

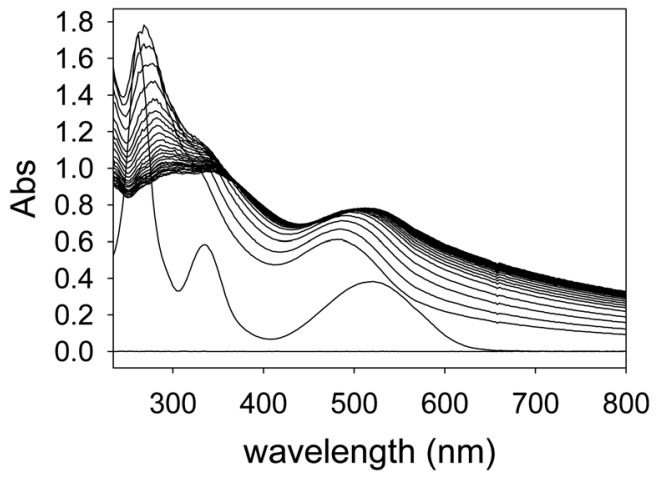

Fig. 6 Changes in the absorption of [PAMAM- $\boldsymbol{m}$-ba.ARS] over the course of $47 \mathrm{~min}$ (spectra acquired every $2 \mathrm{~min}$ ). $T=25^{\circ} \mathrm{C} ; \mathrm{pH}=7.4$ in $50 \mathrm{mM}$ HEPES; $[$ ARS $]=5.10 \times 10^{-5} \mathrm{M}$; [PAMAM- $\boldsymbol{m}$-ba $]=4.50 \times 10^{-5} \mathrm{M}$.

\section{Sugar detection}

Once binding of these dyes to the PAMAM-ba receptors had been confirmed, and conditions to induce these binding events were known, we were in a position to use these receptors and dyes to set up an indicator displacement assay to detect the binding of sugars to these receptors. In fact, since most common sugars do not have a significant intrinsic chromophore or fluorophore, even the direct binding would be spectroscopically silent. Use of an indicator dye in an IDA assay allows us to circumvent that problem, and to continue the use of optical spectroscopy, one of the cheapest, fastest, and easiest to automate instrumental techniques in the analytical chemist's toolbox.

We first determined favourable conditions to prepare each [PAMAM-ba-dye] self-assembled sensor. Previous experience in our group with the construction of IDA sensors suggests using conditions in which the dye is $c a$. $80 \%$ bound to its receptor to optimize responsiveness and sensitivity of the assay. ${ }^{44-46}$

With the above binding data in hand, such conditions were easily determined. D-(-)-Fructose was the first sugar studied: as aliquots of a fructose solution were added to an aqueous solution containing [PAMAM-m-ba-ML] at $\mathrm{pH}$ 7.4, the anisotropy decreased gradually (Fig. 7, left), showing a reversal of the trend observed in the corresponding binding titration (Fig. 1) and indicating that fructose was successfully displacing the ML dye from its complex with PAMAM-m-ba. Although this provided a clearly successful proof of principle, the sensitivity of this assay was not satisfactory: the concentration of fructose had to reach $1 \mathrm{M}$ to induce complete displacement of the dye, suggesting that the [PAMAM-m-ba-ML] complex was not very sensitive to fructose under these conditions.

Comparable results were obtained when trying to displace the ML dye from its complex with the PAMAM-o-ba receptor using fructose at $\mathrm{pH}$ 7.4: full dye displacement could be attained at a comparable or slightly improved concentration of the sugar (ca. 0.5 M fructose, see ESI $\dagger$ ). Additionally, an attempt to sense galactose using the [PAMAM-m-ba-ML] complex was unsuccessful under these conditions: very little dye displacement was observed even at high concentrations of this sugar. In short, the sensitivity of either receptor to sugars was found to be regrettably low under these conditions. 

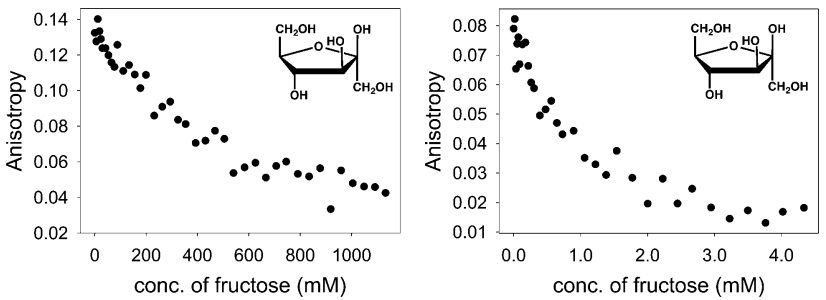

Fig. 7 pH effects: (left) fructose displaces $M L$ from [PAMAM- $\boldsymbol{m}$-ba.ML] at $\mathrm{pH} 7.4$ (50 mM HEPES); (right) the same experiment at $\mathrm{pH} 10.0(50 \mathrm{mM}$ CAPS). The affinity for fructose is much improved at higher $\mathrm{pH} . T=25^{\circ} \mathrm{C}$; $[M L]=1.057 \times 10^{-5} \mathrm{M} ;[$ PAMAM- $\boldsymbol{m}-$ ba $]=1.737 \times 10^{-5} \mathrm{M} ; \lambda_{\mathrm{exc}}=384 \mathrm{~nm}$; $\lambda_{\mathrm{em}}=450 \mathrm{~nm}$.

The effect of $\mathbf{p H}$. Previous studies suggest that boronic acid moieties might display higher affinity for sugars at basic $\mathrm{pH},{ }^{20}$ so, in an attempt to increase the sensitivity of our system, we repeated the displacement experiment with fructose at increased $\mathrm{pH}$. In particular we concentrated on $\mathrm{pH} 10.0$, since excellent results have been reported before for isolated boronate receptors under these conditions. ${ }^{47}$ in the following studies stable buffering was achieved using the well-known CAPS buffer at the same concentration of buffer use in the previous experiments (i.e. $50 \mathrm{mM}$ ). The results were extremely positive (Fig. 7): at $\mathrm{pH} 10$ millimolar concentrations of fructose were sufficient to form a [PAMAM- $\boldsymbol{m}$-ba-fructose] complex, as evidenced by the complete displacement of the ML dye from its complex. This entails a staggering three hundred-fold increase in the observed affinity for fructose, which brought this sensing system within range of analytically interesting concentrations.

Encouraged by these results, we expanded the method to other sugars, including D-ribose, D-glucose and D-galactose. The relevant results are shown in Fig. 8 below. It is particularly promising to see that even sugars such as galactose, to which our sensors did not respond well at neutral $\mathrm{pH}$, could be readily sensed in these improved conditions.

The results in Fig. 8 also show that structurally different sugars display varying affinities towards the PAMAM-ba system. We attribute these differences to the structural features of these sugars: for instance, fructose and ribose are known to form a five-membered furanose ring in solutions, which contain a rigid cis-diol moiety that is known to bind to boronic acid very efficiently. ${ }^{48}$ On the other hand, the pyranose rings prevalent in glucose and galactose in aqueous solution do not contain an equally rigid interaction site; the energetic cost that must be paid to freeze their higher conformational freedom upon binding therefore results in markedly lower affinities observed here.

Similar displacement experiments were also performed on sensing complexes containing the ARS dye (Fig. 9, right). As already mentioned above, anisotropy measurements with ARS complexes were unfortunately hampered by the formation of a small quantity of highly scattering aggregates and were not informative. We monitored the fluorescence emission intensity signal instead. The emission signal was normalized to that of the bound dye: a decrease in the normalized emission indicates dye displacement and therefore sugar binding. Although the
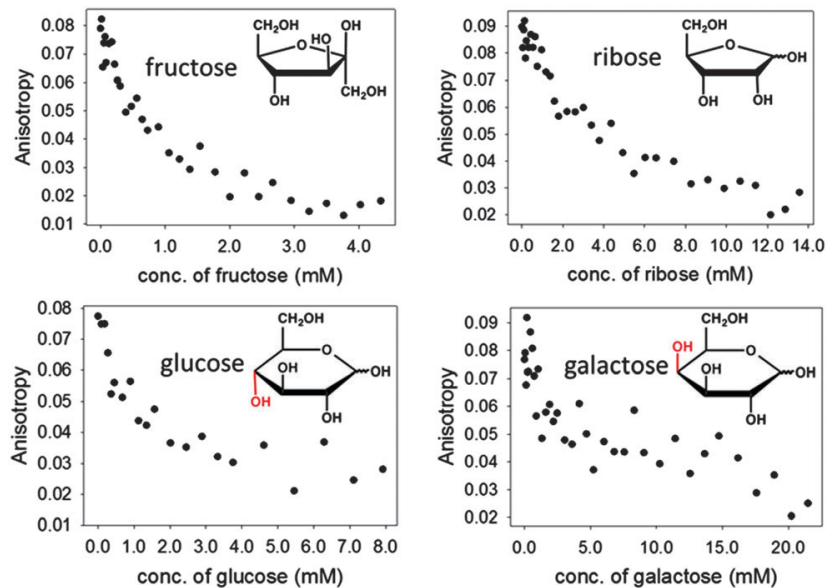

Fig. 8 Sugar binding monitored by the changes in fluorescence anisotropy associated with the displacement of $\mathbf{M L}$ dye from a [PAMAM- $\boldsymbol{m}$-ba.ML] complex at $\mathrm{pH} 10$ in water (50 mM CAPS). $T=25^{\circ} \mathrm{C} ;[\mathrm{ML}]=1.057 \times$ $10^{-5} \mathrm{M}$; [PAMAM- $\boldsymbol{m}$-ba] $=1.737 \times 10^{-5} \mathrm{M} ; \lambda_{\mathrm{exc}}=384 \mathrm{~nm} ; \lambda_{\mathrm{em}}=450 \mathrm{~nm}$. Insets: chemical structures and names of the simple sugars studied.

use of an ARS complex is complicated by the aggregation process described above, it provides an improved dynamic range to the sensing process, since the spectroscopic difference between the dye's free and bound states is more marked.

\section{Discrimination of sugars through array sensing}

The experiments shown above have established that the PAMAM-ba modified dendrimers can act as ligands for carbohydrate moieties; in combination with appropriate dyes, the sugar concentration stimulus can be translated into an optical response by the system.

However, the comparison of sugar responses summarized in Fig. 9 for [PAMAM- $\boldsymbol{m}$-ba-ML] and [PAMAM- $\boldsymbol{m}$-ba-ARS] clearly indicates that neither of these sensors is very selective for any one of the sugars under study: although each sensor responds to the sugar analytes, different analytes cause similar responses and discrimination based on any single analytical signal (i.e. a univariate approach) would be unsuccessful.

For instance, the largest difference between all the sugars in the [PAMAM- $\boldsymbol{m}$-ba-ML] system was observed at around a $2 \mathrm{mM}$ analyte concentration; however, fructose could not be differentiated from glucose. On the other hand, the sensor using the
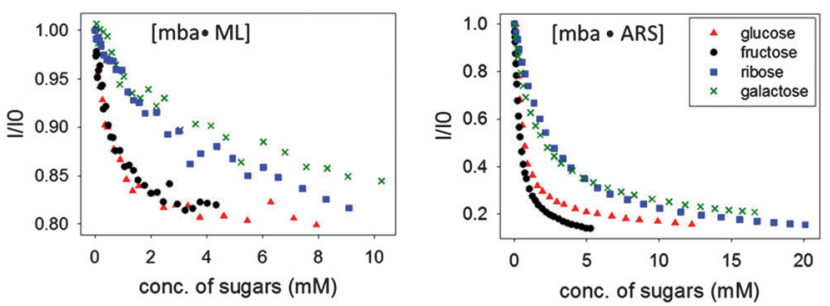

Fig. 9 Normalized fluorescence intensity changes associated with dye displacement from [PAMAM- $\boldsymbol{m}$-ba.ML] (left) and [PAMAM- $\boldsymbol{m}$-ba.ARS] (right). $T=25{ }^{\circ} \mathrm{C}$; $\mathrm{pH}=10.0$ in water $(50 \mathrm{mM}$ CAPS); For $\mathrm{ML}$ : $[\mathrm{ML}]=$ $1.057 \times 10^{-5} \mathrm{M}$; [PAMAM- $\boldsymbol{m}$-ba] $=1.737 \times 10^{-5} \mathrm{M} ; \lambda_{\mathrm{exc}}=384 \mathrm{~nm} ; \lambda_{\mathrm{em}}=$ $450 \mathrm{~nm}$. For ARS: [ARS] $=1.057 \times 10^{-5} \mathrm{M}$; [PAMAM- $\boldsymbol{m}$-ba] $=2.323 \times 10^{-5}$ $\mathrm{M} ; \lambda_{\mathrm{exc}}=500 \mathrm{~nm} ; \lambda_{\mathrm{em}}=570 \mathrm{~nm}$. 
ARS dye was able to discriminate between glucose and fructose under those conditions, but not between ribose and galactose. In short, the combined results obtained from both sensors were found to contain sufficient information to effect differentiation. In order to harvest this information effectively, we use these two sensing systems to construct a cross-reactive array sensing system to sense and discriminate different sugars. ${ }^{49,50}$ Using array sensing techniques our group has recently reported on the recognition of e.g. physiologically relevant phosphate esters, ${ }^{46}$ organophosphates including pesticides and nerve gas agents, ${ }^{45}$ and divalent metal ions. ${ }^{44}$

Since each one of the four sugars tested so far has a slightly different interaction with each [PAMAM-ba-dye] complex, a unique response pattern for each certain sugar can be generated from which that sugar can be recognized (Scheme 5).

In the present case we constructed a sensing array by using the following building blocks: $2 \times$ receptors, PAMAM- $\boldsymbol{m}$-ba and PAMAM-o-ba; $2 \times$ dyes (ARS and ML); $2 \times$ pH conditions $(\mathrm{pH} 7.4$ and 10.0). Each receptor-dye-condition combination represented one component of the sensing array. Each component was exposed to the four sugars in turn $([$ sugar $]=2.00 \mathrm{mM}$; ARS $]=0.0514 \mathrm{mM}$; $[\mathbf{M L}]=0.0103 \mathrm{mM} ;[$ PAMAM- $\boldsymbol{m}$-ba $]=0.0174 \mathrm{mM} ;[$ PAMAM-o-ba $]=$ $0.0102 \mathrm{mM}$ ).

Array responses were measured by monitoring absorption, and fluorescence intensity for both ML and ARS complexes, and fluorescence anisotropy for the ML sensor (anisotropy for the ARS sensor was not available as discussed above); a total of 9 experimental measurements were acquired per each sample replicate. Twelve replicates were measured for each analyte. Samples were laid out on 384-well plates together with relevant blanks and references. All measurements were then carried out on a multimode microwell plate reader capable of acquiring absorbance, fluorescence emission intensity and polarization; the use of a microwell plate reader was crucial to the development of the assay, as it allowed for automated and highly repeatable

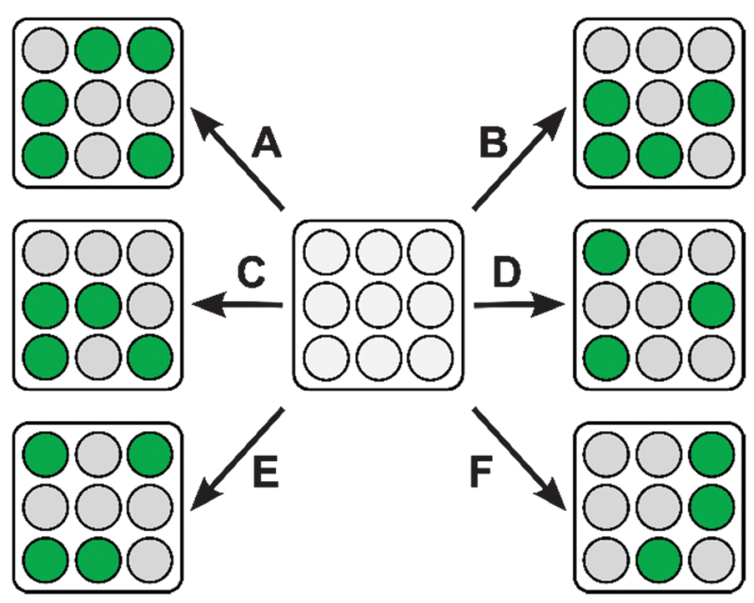

Scheme 5 A pattern-based sensing array. The array, composed of multiple receptors (centre) is exposed to each analyte in turn (A-F). Sensors in the array are not selective for any of the analytes, but the overall response of the whole array generates a unique and recognizable "finger print" of each analyte, which allows for selective recognition. multivariate data acquisition. Plate layouts and further experimental details are reported in the ESI. $\dagger$

Once raw experimental measurements were acquired, we used linear discriminant analysis (LDA) to analyse the data set. LDA has long been used for complex data reduction in applications such as face and voice recognition $;{ }^{51}$ more recently, it has also been introduced to the chemometrics community. ${ }^{52,53}$ The purpose of the multivariate LDA algorithm is to generate linear combinations of the original raw measurements with the purpose of minimizing the dispersion between replicate measurements of each analyte, while at the same time maximizing the distance between analyte clusters. In doing so, LDA generates a new set of variables (factors), returned in the order of decreasing information content. By choosing the two most informationrich factors and discarding others that contributed only marginally to the differentiation, the dimensions of the data set were drastically reduced; data points were then plotted on a two dimensional graph according to their scores along each LDA factor.

The ultimate results of data processing on the samples obtained from our sensing array are shown in Fig. 10 as an LDA score plot. Replicate samples corresponding to the same sugar were found to cluster close together while at the same time clusters of samples corresponding to different sugars were found to be well separated from each other. The score plot reported here represents $97.9 \%$ of the total information content that was available in the raw data set, indicating that data reduction could be carried out with only minimal information loss.

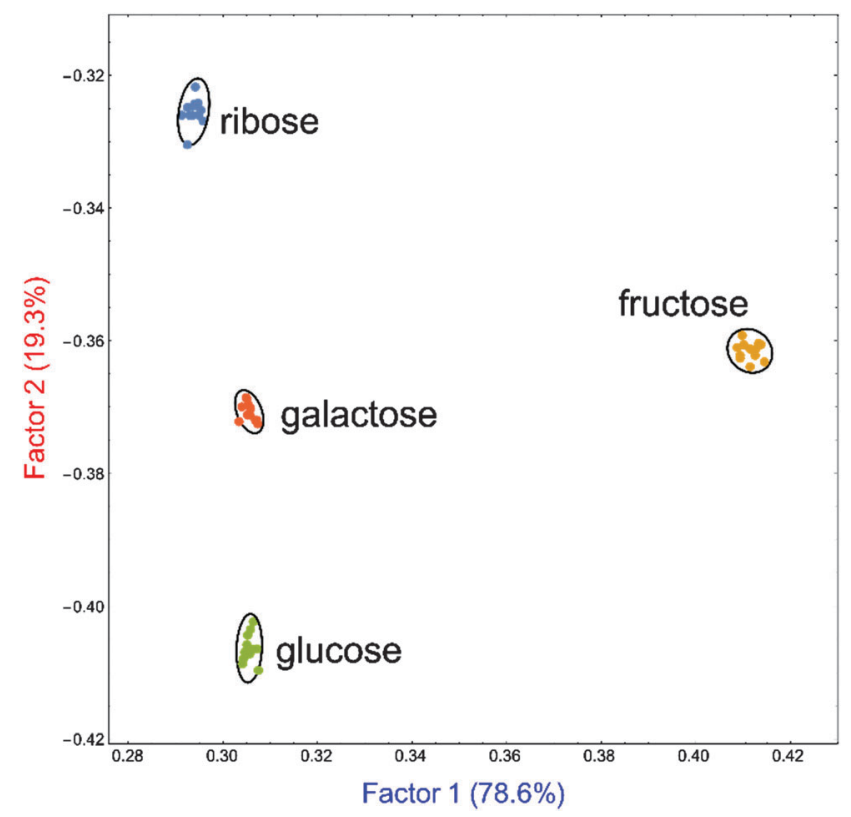

Fig. 10 Two-dimensional score plot of the results from linear discriminant analysis (LDA) on the dataset obtained by exposing a sensing array containing two PAMAM-ba sensors, two dyes (ARS and $\mathbf{M L}$ ), and two $\mathrm{pH}$ conditions $(\mathrm{pH} 7.4$ and 10.0) to four simple sugars (glucose, galactose, ribose, fructose). The percentages reported on each axis title refer to the fraction of the information from the initial dataset that is represented by that axis. This score plot represents a total of $(78.6+19.3) \%=97.9 \%$ of the overall information content in the raw dataset: data reduction was carried out with minimal information loss. 
In short, the LDA plot in Fig. 10 demonstrated that we were able to use a series of poorly selective self-assembled sensors based on PAMAM-m-ba and PAMAM-o-ba and two dyes to construct a sensing array capable of qualitative differentiation of simple sugars in neat water solution. Of particular interest is the ability of this sensor to discriminate between very similar structures such as glucose and galactose, which differ only by the configuration of a single chiral centre in their structure.

\section{Conclusions}

Two new sugar receptors, PAMAM- $\boldsymbol{m}$-ba and PAMAM-o-ba, were synthesized. The binding properties of these receptors and dyes 4-methylesculetin (ML) and alizarin red S (ARS) were studied by fluorescence and UV-Vis spectroscopy in neutral aqueous solution. As desired, PAMAM- $\boldsymbol{m}$-ba and PAMAM-o-ba displayed excellent solubility in water, much improved from that of the corresponding isolated boronic acid. Both PAMAM-m-ba and PAMAM-o-ba bound to ML and ARS dyes with high affinity in aqueous solution, which opened the door for the construction of indicator displacement assays for the sensing of sugars in neat water.

Displacement experiments with sugars were performed successfully at pH 7.4 and at pH 10.0. These sensors displayed greatly improved affinity for simple sugars even in neat water, typically a very challenging medium for sugar recognition and sensing. The highest affinity was observed at around $\mathrm{pH}$ 10.0: under the latter conditions, this sensing system was also sensitive to glucose, galactose and ribose. In conclusion, our initial hypothesis that dendritic boronic acid derivatives would display much improved affinity towards sugars when compared to their isolated counterparts was proved correct.

As a proof of principle of possible applications of these receptors and because the selectivity of the individual receptors was found to be poor under these conditions, we constructed a pattern-based sensing array using multiple combinations of the two receptors (PAMAM-m-ba and PAMAM-o-ba), two dyes (ARS and $\mathrm{ML}$ ), and two $\mathrm{pH}$ conditions (7.4 and 10.0) outlined above. The high-dimensionality data obtained from this array were analysed using multivariate techniques, resulting in excellent clustering results: replicate samples for the same sugar were clustered tightly, while data clusters from different sugars were well-separated. As a proof of principle, the system we propose here is therefore capable of qualitative recognition and differentiation of fructose, ribose, glucose, and galactose, the latter two being particularly impressive feats given their great structural similarity.

\section{Acknowledgements}

We gratefully acknowledge the help of Dr Yuping Bao and her group in the Department of Chemical Engineering at The University of Alabama for use of their instrument and their help in carrying out the dynamic light scattering experiments. We also acknowledge the financial support from the Department of Chemistry at The University of Alabama (XL), and from the University of Alabama Faculty Start-up Funds (MB).

\section{References}

1 X. Sun, W. Zhai, J. S. Fossey and T. D. James, Chem. Commun., 2016, 52, 3456-3469.

2 K. Ariga, H. Ito, J. P. Hill and H. Tsukube, Chem. Soc. Rev., 2012, 41, 5800-5835.

3 N. Y. Edwards, T. W. Sager, J. T. McDevitt and E. V. Anslyn, J. Am. Chem. Soc., 2007, 129, 13575-13583.

4 M. Mazik, Chem. Soc. Rev., 2009, 38, 935-956.

5 C. D. Geddes and J. R. Lakowicz, Glucose sensing, Springer, New York, NY, 2006.

6 T. E. Curey, A. Goodey, A. Tsao, J. Lavigne, Y. Sohn, J. T. McDevitt, E. V. Anslyn, D. Neikirk and J. B. Shear, Anal. Biochem., 2001, 293, 178-184.

7 C. C. Chen, V. Llado, K. Eurich, H. T. Tran and E. Mizoguchi, Clin. Immunol., 2011, 140, 268-275.

8 N. Kawashima, S. J. Yoon, S. Hakomori and K. Nakayama, Glycobiology, 2006, 16, 1117-1118.

9 L. D. Carrillo, L. Krishnamoorthy and L. K. Mahal, J. Am. Chem. Soc., 2006, 128, 14768-14769.

10 M. Ramon, F. Rolland and J. Sheen, The Arabidopsis Book, American Society of Plant Biologists, 2008, vol. 6, p. e0117.

11 S. Shinkai and M. Takeuchi, Trends Anal. Chem., 1996, 15, 188-194.

12 X. Wu, Z. Li, X. X. Chen, J. S. Fossey, T. D. James and Y. B. Jiang, Chem. Soc. Rev., 2013, 42, 8032-8048.

13 G. V. Oshovsky, D. N. Reinhoudt and W. Verboom, Angew. Chem., Int. Ed., 2007, 46, 2366-2393.

14 S. Arimori, M. L. Bell, C. S. Oh and T. D. James, Org. Lett., 2002, 4, 4249-4251.

15 T. D. James, K. R. A. Samankumara Sandanayake and S. Shinkai, Nature, 1995, 374, 345-347.

16 Y. Egawa, R. Miki and T. Seki, Materials, 2014, 7, 1201-1220.

17 Y. Egawa, R. Gotoh, T. Seki and J.-i. Anzai, Mater. Sci. Eng., C, 2009, 29, 115-118.

18 H. Eggert, J. Frederiksen, C. Morin and J. C. Norrild, J. Org. Chem., 1999, 64, 3846-3852.

19 W. Yang, H. He and D. G. Drueckhammer, Angew. Chem., Int. Ed., 2001, 40, 1714-1718.

20 G. Springsteen and B. Wang, Tetrahedron, 2002, 58, 5291-5300.

21 R. M. Crooks and A. J. Ricco, Acc. Chem. Res., 1998, 31, 219.

22 R. Esfand and D. A. Tomalia, Drug Discovery Today, 2001, 6, 427.

23 D. Cakara, J. Kleimann and M. Borkovec, Macromolecules, 2003, 36, 4201.

24 Y. Niu, L. Sun and R. M. Crooks, Macromolecules, 2003, 36, 5725. 25 T. J. Prosa, B. J. Bauer and E. J. Amis, Macromolecules, 2001, 34, 4897-4906.

26 X. Zhang, L. You, E. V. Anslyn and X. Qian, Chemistry, 2012, 18, 1102-1110.

27 S. H. Shabbir, C. J. Regan and E. V. Anslyn, Proc. Natl. Acad. Sci. U. S. A., 2009, 106, 10487-10492. 
28 L. Zhu, Z. L. Zhong and E. V. Anslyn, J. Am. Chem. Soc., 2005, 127, 4260-4269.

29 G. Springsteen and B. Wang, Chem. Commun., 2001, 1608-1609.

30 For instance, see Dendritech Inc., http://www.dendritech. com whose products are also distributed by Sigma-Aldrich.

31 F. Chen, K. Wu, Y. Liu, H. Huang and K. He, J. Polym. Eng., 2014, 34, 733-738.

32 B. Menot, J. Stopinski, A. Martinez, J.-B. Oudart, F.-X. Maquart and S. Bouquillon, Tetrahedron, 2015, 71, 3439-3446.

33 Z. Wang, C. Chen, R. Liu, A. Fan, D. Kong and Y. Zhao, Chem. Commun., 2014, 50, 14025-14028.

34 T. D. James, H. Shinmori, M. Takeuchi and S. Shinkai, Chem. Commun., 1996, 705-706.

35 S. Shinkai and A. Robertson, The design of molecular artificial sugar sensing systems, Springer-Verlag, 2001.

36 T. D. James, K. R. A. Sandanayake Samankumara and S. Shinkai, Angew. Chem., Int. Ed., 1996, 35, 1911-1922.

37 S. W. Chaikin and W. G. Brown, J. Am. Chem. Soc., 1949, 71, 122-125.

38 M. Hudlicky, Reductions in organic chemistry, American Chemical Society, Washington, DC, 2nd edn, 1996.

39 M. R. Naimi-Jamal, J. Mokhtari, M. G. Dekamin and G. Kaupp, Eur. J. Org. Chem., 2009, 3567-3572.

40 J. R. Lakowicz, Principles of fluorescence spectroscopy, Springer, New York, 3rd edn, 2006.
41 I. Willerich, T. Schindler, H. Ritter and F. Groehn, Soft Matter, 2011, 7, 5444-5450.

42 I. Willerich, Y. Li and F. Gröhn, J. Phys. Chem. B, 2010, 114, 15466.

43 I. Willerich and F. Groehn, J. Am. Chem. Soc., 2011, 133, 20341-20356.

44 A. M. Mallet, A. B. Davis, D. R. Davis, J. Panella, K. J. Wallace and M. Bonizzoni, Chem. Commun., 2015, 51, 16948-16951.

45 Y. L. Liu and M. Bonizzoni, J. Am. Chem. Soc., 2014, 136, 14223-14229.

46 A. M. Mallet, Y. Liu and M. Bonizzoni, Chem. Commun., 2014, 50, 5003-5006.

47 J. Yan, G. Springsteen, S. Deeter and B. Wang, Tetrahedron, 2004, 60, 11205-11209.

48 S. Jin, Y. Cheng, S. Reid, M. Li and B. Wang, Med. Res. Rev., 2010, 30, 171-257.

49 K. L. Diehl and E. V. Anslyn, Chem. Soc. Rev., 2013, 42, 8596-8611.

50 J. J. Lavigne and E. V. Anslyn, Angew. Chem., Int. Ed., 2001, 40, 3118-3130.

51 J. Lu and K. N. Plataniotis, in IEEE Transactions on Neural Networks, The Institute of Electrical and Electronics Engineers (IEEE), 2003, vol. 14, pp. 195-201.

52 M. Otto, Chemometrics: statistics and computer application in analytical chemistry, Wiley-VCH, Weinheim, New York, 1999.

53 R. G. Brereton, Chemometrics for pattern recognition, Wiley Interscience, Chichester, U.K., 2009. 